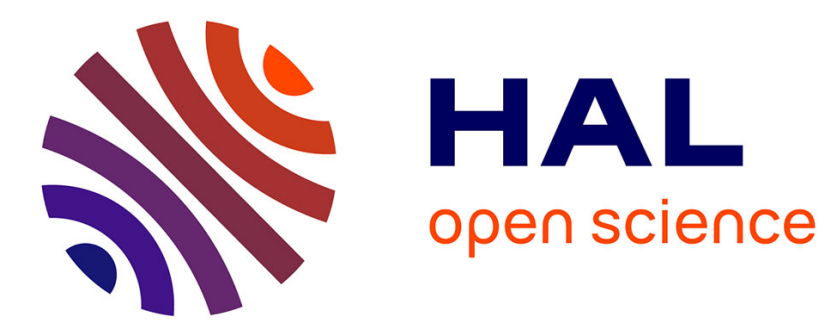

\title{
Blind MRI Brain Lesion Inpainting Using Deep Learning
}

José V Manjón, Jose E Romero, Roberto Vivo-Hernando, Gregorio Rubio, Fernando Aparici, Maria de La Iglesia-Vaya, Thomas Tourdias, Pierrick Coupé

\section{To cite this version:}

José V Manjón, Jose E Romero, Roberto Vivo-Hernando, Gregorio Rubio, Fernando Aparici, et al.. Blind MRI Brain Lesion Inpainting Using Deep Learning. International Workshop on Simulation and Synthesis in Medical Imaging, Oct 2020, LIMA, Peru. pp.41-49, 10.1007/978-3-030-59520-3_5 . hal-02966536

\section{HAL Id: hal-02966536 \\ https://hal.science/hal-02966536}

Submitted on 14 Oct 2020

HAL is a multi-disciplinary open access archive for the deposit and dissemination of scientific research documents, whether they are published or not. The documents may come from teaching and research institutions in France or abroad, or from public or private research centers.
L'archive ouverte pluridisciplinaire HAL, est destinée au dépôt et à la diffusion de documents scientifiques de niveau recherche, publiés ou non, émanant des établissements d'enseignement et de recherche français ou étrangers, des laboratoires publics ou privés. 


\title{
Blind MRI Brain lesion inpainting using Deep Learning
}

José V. Manjón ${ }^{1}$, José E. Romero ${ }^{1}$, Roberto Vivo-Hernando ${ }^{2}$, Gregorio Rubio ${ }^{3}$, Fernando Aparici ${ }^{4}$, Maria de la Iglesia-Vaya ${ }^{5}$, Thomas Tourdias ${ }^{6}$ and Pierrick Coupé ${ }^{7}$

${ }^{1}$ Instituto de Aplicaciones de las Tecnologías de la Información y de las Comunicaciones Avanzadas (ITACA), Universitat Politècnica de València, Camino de Vera s/n, 46022 Valencia, Spain

${ }^{2}$ Instituto de Automática e Informática Industrial, Universitat Politècnica de València, Camino de Vera s/n, 46022 Valencia, Spain.

${ }^{3}$ Departamento de matemática aplicada, Universitat Politècnica de València, Camino de Vera s/n, 46022 Valencia, Spain.

${ }^{4}$ Área de Imagen Médica. Hospital Universitario y Politécnico La Fe. Valencia, Spain

${ }^{5}$ Brain Connectivity Laboratory, Joint Unit FISABIO \& Prince Felipe Research Centre (CIPF), Valencia, Spain

${ }^{6} \mathrm{CHU}$ de Bordeaux, Service de neuroimagerie diagnostique et thérapeutique, F-33076, Bordeaux, France

${ }^{7}$ CNRS, LaBRI, UMR 5800, PICTURA, F-33400 Talence, France

\begin{abstract}
In brain image analysis many of the current pipelines are not robust to the presence of lesions which degrades their accuracy and robustness. For example, performance of classic medical image processing operations such as non-linear registration or segmentation rapidly decreases when dealing with lesions. To minimize their impact, some authors have proposed to inpaint these lesions so classic pipelines can be used. However, this requires to manually delineate the regions of interest which is time consuming. In this paper, we propose a deep network that is able to blindly inpaint lesions in brain images automatically allowing current pipelines to robustly operate under pathological conditions. We demonstrate the improved robustness/accuracy in the brain segmentation problem using the SPM12 pipeline with our automatically inpainted images.
\end{abstract}

Keywords: Lesion inpainting, MRI, deep learning, robust segmentation.

\section{Introduction}

Most of state-of-the-art brain image analysis packages have been designed to work with anatomically consistent images using some assumptions such as the existence of a fixed number of expected tissues for example. Therefore, when the images have pathological alterations such as lesions, these assumptions are violated leading to biased/incorrect results. Although the development of more complex pipelines covering a wider set of conditions could manage this problem, we are also interested by using well-established standard packages such as Freesurfer or SPM (for example for longitudinal analysis). 
One possible solution to this problem has been already suggested by several authors in the past, image inpainting. Image inpainting [1] refers to the process where specific regions of an image or volume are masked-out and filled with plausible information from the image itself or other "similar" images. In medical imaging, this technique has been used, for example, to fill multiple sclerosis (MS) lesions with white matter consistent intensities resulting in realistic healthy-looking versions of the images [24]. Guizard et al. [3] filled the lesion regions with locally consistent intensities using a patch-based multi-resolution onion peel strategy. More recently, Prados et al. [4] proposed a "modality-agnostic" patch-based method for lesion filling also based on the non-local means strategy. Using deep learning, Armanious et al. [5] recently proposed a 2D network to inpaint medical image modalities using an adversarial approach.

All these inpainting methods require the definition of the regions to inpaint. This definition can be performed manually (which is a time consuming, tedious and error prone procedure) or automatically using a lesion segmentation algorithm. Unfortunately, segmentation-based approaches highly depend on the quality of the segmentation (especially the number of false negatives).

An alternative where there is no need to explicitly define the regions to inpaint is called blind inpainting [6]. Blind inpainting behaves as a restoration process where the input image is considered as "corrupted" and the method tries to directly predict the "clean" image by learning the relationship between them. For example, Liu et al. [6] used a Residual Deep network to predict the clean image without any specific definition on the regions to fill.

In this paper, we propose, as far as we know, the first 3D blind inpainting method in medical imaging. We propose a deep network which is able to predict a "clean" image from an image containing abnormal areas (lesions). To construct our network, we split the process into two steps. We first learn how to inpaint anatomically normal images (with random regions masked out) and we applied it to reconstruct MS images masking out the lesions to obtain their healthy versions. In the second step, we use these healthy-looking images as output of a network feed with their corresponding unhealthy version. We show the benefits of our inpainting method on segmentation problem but its application is not limited to this problem as it can help in other problems such as longitudinal non-linear registration for example.

\section{$2 \quad$ Material and Methods}

\subsection{Datasets}

We used three different datasets, one containing cases with no anatomical abnormalities (lesions) and two with multiple sclerosis (MS) lesions.

Normal dataset without lesions: The open access IXI dataset (https://braindevelopment.org/ixi-dataset/) was used. This dataset contains images of 580 healthy 
subjects from several hospitals in London (UK). Both $1.5 \mathrm{~T}$ and $3 \mathrm{~T}$ images were included in our training dataset. 3T images were acquired on a Philips Intera 3T scanner $\left(\mathrm{TR}=9.6 \mathrm{~ms}, \mathrm{TE}=4.6 \mathrm{~ms}\right.$, flip angle $=8^{\circ}$, slice thickness $=1.2 \mathrm{~mm}$, volume size $=256 \times 256 \times 150$, voxel dimensions $=0.94 \times 0.94 \times 1.2 \mathrm{~mm} 3) .1 .5 \mathrm{~T}$ images were acquired on a Philips Gyroscan 1.5T scanner $(\mathrm{TR}=9.8 \mathrm{~ms}$, $\mathrm{TE}=4.6 \mathrm{~ms}$, flip angle $=8^{\circ}$, slice thickness $=1.2 \mathrm{~mm}$, volume size $=256 \times 256 \times 150$, voxel dimensions $=0.94 \times 0.94 \times 1.2 \mathrm{~mm} 3$ ). Images from subjects presenting visual lesions or severe image artifacts were excluded from selection, being the final number of subjects used of 298 .

MS dataset with manual segmentation of MS lesions: Our MS dataset is composed of 3T 3D-T1w MPRAGE images from 43 patients previously used in the development of lesionBrain pipeline [7]. These images were preprocessed to align them into the MNI space and to normalize their intensities. Manual segmentations of white matter lesions were obtained as described in [7].

MS dataset with automatic segmentation of MS lesions: This dataset consist of 200 cases automatically analyzed using lesionBrain segmentation pipeline within the volBrain online platform. These $\mathrm{T} 1+$ lesion masks were visually checked to assess their quality.

\subsection{Pipeline description}

Preprocessing: First, the images are preprocessed to normalize their intensities and to register them into the MNI space (1 $\mathrm{mm}^{3}$ voxel resolution). A denoising step based on the adaptive nonlocal means filter is first applied [8]. Next, the images are registered into the MNI space using an affine transform [9]. Finally, inhomogeneity correction is performed [10]. Intensity standardization was performed dividing the images by their mean.

Inpainting network topology: The proposed network is a 3D UNet-like network [11] (See Fig. 1), which learns a non-linear mapping between the source image (image to inpaint) and target image (inpainted image). To work with the full 3D volume, due to memory constraints, we decomposed the input volume into 8 sub-volumes using a 3D spatial-to-depth decomposition by decimating the original volume with one voxel shift in each 3D dimension. These 8 sub-volumes were structured as an input tensor of $91 \times 109 \times 91 \times 8$ containing all the voxels of the original input volume.

In our 3D UNET, the encoder path consists of a 3D convolution layer (kernel size of $3 \times 3 \times 3$ voxels) for each resolution level with ReLU activation and batch normalization layers followed by a max pooling layer and upsampling layer is used in the decoder path. The first resolution level used 40 filters with an increment of factor 2 in the subsequent lower resolution levels in order to balance the loss of spatial resolution. Similarly, the number of filters is decreased by 2 in the decoder path at sub- 
sequent resolution levels. We added a final reconstruction block consisting in a trilinear interpolation layer followed by a 3D convolution layer (with 8 filters) plus a

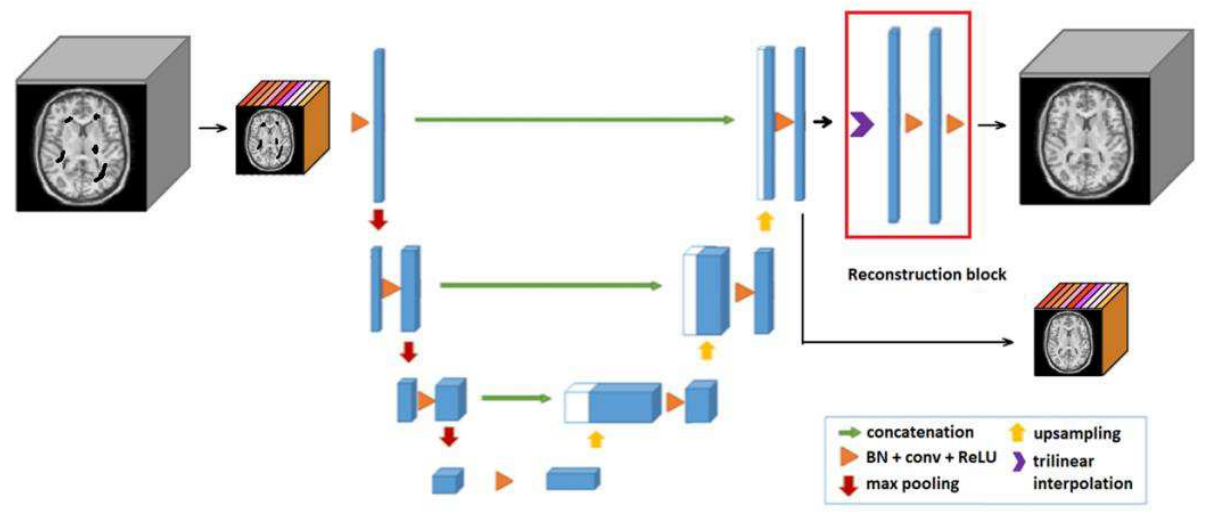

Figure 1. Scheme of the proposed 3D network for MRI inpainting.

ReLU and Batch normalization layers. Finally, a last 3D convolution layer with a single filter of size $1 \times 1 \times 1$ was used to produce the full resolution 3D output (see Fig. 1 for more details).

To train the network, we used the mean squared error loss and a deep supervision approach [12]. The first output is the low-resolution depth-wise output and the second the full resolution volume after the reconstruction block (see figure 1).

Non-blind vs blind inpainting: In classic inpainting procedures one or several regions are marked out in the image as areas to be filled with "realistic" data from other images or the image itself. Therefore, this requires the explicit manual definition of these areas which is time consuming and error prone. In this paper, we propose a deep convolution neural network for blind WM lesion inpainting that does not require the manual definition of lesions. To obtain this network, we split the process in two steps: i) generation of synthetic training data using a non-blind inpainting network and ii) the development of a blind inpainting network.

1. Development of a non-blind inpainting network. In order to generate training dataset for the blind inpainting network described in the following, we first train a non-blind inpainting network able to reconstruction healthy looking image from masked-out images. To train this network, we used the architecture previously defined. As training data, we used as input randomly selected normal anatomy cases $(\mathrm{N}=298)$ masked out with randomly selected lesion masks $(\mathrm{N}=500)$ automatically generated using lesionBrain (which includes large, medium and small lesion load examples). Note that these lesion masks were different from the masks of the MS dataset with automatic lesion segmentation. As output, we used the original unmasked images. We masked 
out the regions to inpaint by setting those pixels to -1 to clearly differentiate them from the pixels that have to remain unchanged that are positive defined.

2. Development of a blind inpainting network: To train this network, we used the same architecture but with different training data. As input data, we used automatically segmented MS images without any masking $(\mathrm{N}=200)$. Since no lesion-free version of our MS cases is available for the output, we used as a surrogate their inpainted versions obtained by applying the previously trained non-blind inpainting network masking out the lesions using the available automatic segmentation. To teach the network also when not to inpaint (healthy cases) we added as input/output images from IXI dataset. In this case the network just behaves as an autoencoder. This lets the network to be blindly applied seamlessly to either healthy or lesioned cases.

\section{$3 \quad$ Results}

All the experiments were performed using Tensorflow 1.15 and Keras 2.2.4 in a Titan $\mathrm{X}$ GPU with $12 \mathrm{~GB}$ of memory. To train the network we used an Adam optimizer with default parameters. A data generator function was used to feed the network during 200 epochs (50 cases per epoch). To measure the performance of the proposed method, we used two different metrics providing information from different perspectives, the peak-signal-to-noise ratio (PSNR) and the correlation coefficient (all computed over the whole volume).

\section{Reconstruction performance}

First, we trained the non-blind inpainting network. We randomly selected from the normal dataset 258 cases for training, 20 for validation and 20 for test. We used data augmentation within the generator consisting of left-right flipping the input and output randomly. The test PSNR was 48.13 vs. 34.21 of the input images and the correlation was almost perfect 0.9997 compared to the original input image correlation (0.9835). Moreover, a careful visual inspection corroborated the successful recovery of WM areas. Therefore, we considered our first non-blind inpainting network able to reconstruct healthy-looking images from masked-out images. This non-blind network is then used to generate synthetic training data for the blind inpainting network. Consequently, we applied this network to the automatically segmented MS dataset to generate the corresponding lesion-free images. Again, a visual inspection verified the successful generation of the images.

Second, we trained the blind inpainting network using as input the original MS data and as output the inpainted images generated by the first network. This time, we randomly selected 180 cases for training and 20 for validation. The test images were the original 43 manually segmented cases. We also added to the training dataset the 268 normal cases (218 for training, 20 for validation and 20 for test) so the network can 
also learn when to inpaint and when not do it. Again, we used a data augmentation policy within the generator consisting of left-right flipping the input and output randomly. The test PSNR for the blind network was 49.57 for MS cases and 44.97 for the normal images. The correlation of both test MS cases (0.9998) and normal cases (0.9999) was again very high.

From the efficiency point of view, the proposed method has a computational time of 0.5 seconds when running in GPU and around 8 seconds in CPU.

\section{Impact on segmentation}

To evaluate the impact of the inpainting process in the segmentation of brain tissues on normal subjects and MS patients, we used the well-known SPM12 software [13]. SPM12 uses a generative model to segment the brain into different tissues such as grey matter (GM), white matter (WM) and cerebrum-spinal fluid (CSF) among others. Although SPM12 uses a powerful Bayesian approach including spatial tissue priors to help in the classification process, it normally fails when WM lesions are present, incorrectly labeling them as GM. Eventually, these lesions can be masked out to avoid problems, but this requires their manual or automatic delineation. Alternatively, we propose to segment the blindly inpainted images.

We segmented the non-blind inpainted (first network) MS test images with SPM12 and we considered them as the ground truth segmentations after checking that they did not include any WM lesion misclassification. Then SPM12 was also applied to the original and blind-inpainted images and their segmentations were compared to the ground truth using the DICE metric. Results are summarized in table 1 and an example result is shown at figure 2 .

Table 1. Mean Dice comparison on test MS data using the proposed blind-inpainting network.*statistically significant differences.

\begin{tabular}{c|cc}
\hline & \multicolumn{2}{|c}{ MS (N=43) } \\
\hline Tissue & Original & Inpainted \\
\hline GM & $0.9729 \pm 0.0158$ & $\mathbf{0 . 9 8 4 5} \pm \mathbf{0 . 0 1 1 5 *}$ \\
WM & $0.9707 \pm 0.0190$ & $\mathbf{0 . 9 8 2 3} \pm \mathbf{0 . 0 1 4 1 *}$ \\
\hline Average & $0.9718 \pm 0.0172$ & $\mathbf{0 . 9 8 3 4} \pm \mathbf{0 . 0 1 2 8}$ \\
\hline
\end{tabular}

As can be noted, the inpainted MS images provided significantly better DICE coefficients in average and for both GM and WM tissues (Wilcoxon signed-rank test). 

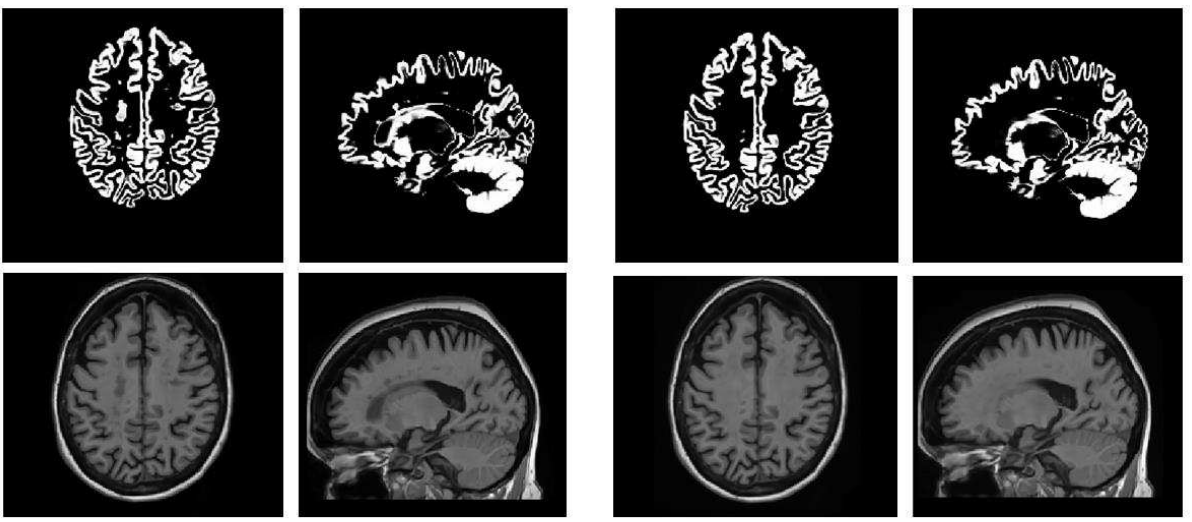

Original

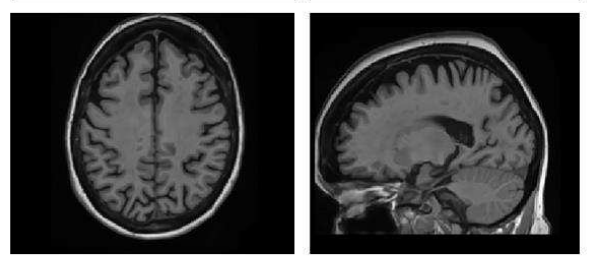

Blindly inpainted

Figure 2. Left: Original MS image and its GM segmentation. Right: Blindly-inpainted image and its GM segmentation (note that most of WM lesions no longer appear as GM).

\section{Abnormal WM segmentation}

Although the inpainted images can be used to robustly classify GM and WM tissues, we might want to label the WM lesions as abnormal WM since this can be an interesting information for some neurological diseases. A simple way to obtain this information is to compute the absolute difference between the original and inpainted images and then select those voxels (within the WM area) with values higher than a fixed threshold $\lambda$. An illustrative example of this approach $(\lambda=0.5)$ is shown in fig. 3 .

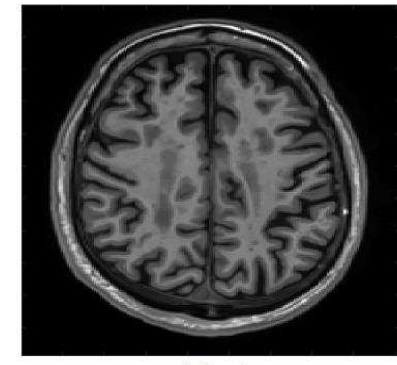

Original

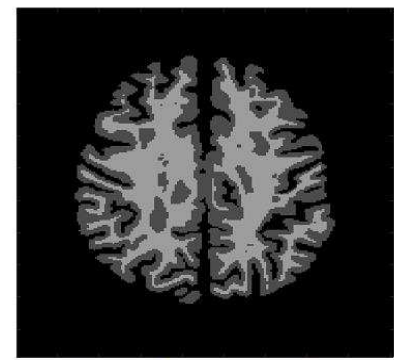

Original segmentation

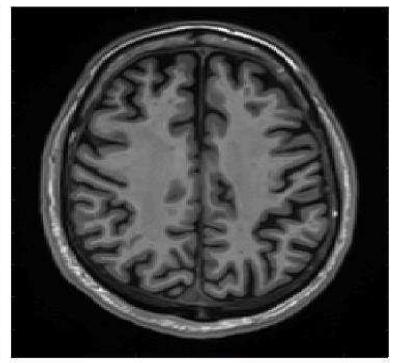

Inpainted

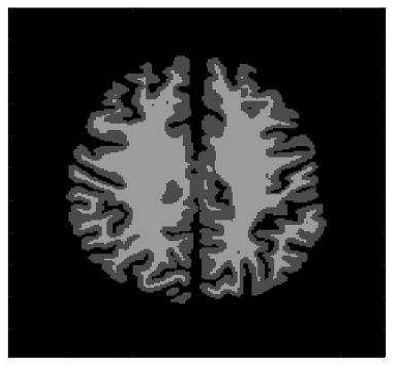

Inpainted segmentation

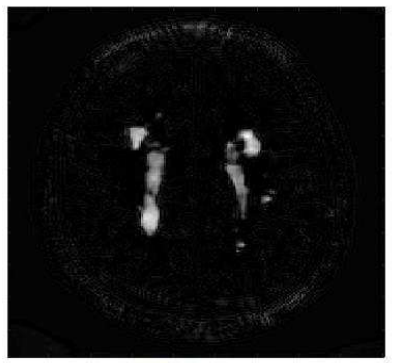

Difference

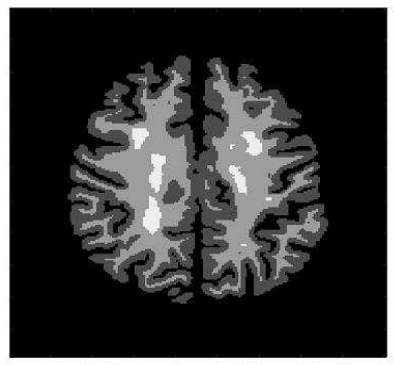

Inpainted segmentation + lesions 
Figure 3. First row: example of abnormal WM segmentation obtained from the image difference between original and inpainted images. Second row: segmentation results.

\section{Conclusion}

In this paper, we have presented a novel MRI 3D inpainting method which is able to blindly remove WM lesions from T1 images. As far as we know, this is the first blind inpainting method proposed in MRI and the first fully 3D approach (previous methods work only in $2 \mathrm{D}$ or $3 \mathrm{D}$ patches). To work with a single volume covering the entire brain (no 3D patch-wise or 2D slice-wise strategies) we used a 3D spatial-todepth decomposition which allows to use a larger number of filters without losing any image resolution. To construct our network, we first learned how to inpaint anatomically normal images and we applied it to reconstruct MS images masking out the lesions to obtain their healthy versions. Finally, our final inpainting network was obtained simply mapping the MS images in their healthy versions. It is worth to note that our proposed method can be applied not only to MS but to any other disease that alters normal brain anatomy (vascular lesions, brain tumors, etc.). This will be explored in the future.

We have shown the benefits of our inpainting method on the segmentation problem using as example SPM12 software where robust tissue segmentation can be obtained (and outlier detection, i.e. lesions). However, its usefulness is not limited to this specific problem as it can help in other problems such as longitudinal non-linear registration for example.

Acknowledgement: This research was supported by the Spanish DPI2017-87743-R grant from the Ministerio de Economia, Industria y Competitividad of Spain. This work also benefited from the support of the project DeepvolBrain of the French National Research Agency (ANR18-CE45-0013). This study was achieved within the context of the Laboratory of Excellence TRAIL ANR-10-LABX-57 for the BigDataBrain project. Moreover, we thank the Investments for the future Program IdEx Bordeaux (ANR-10- IDEX- 03- 02, HL-MRI Project), Cluster of excellence CPU and the CNRS. The authors gratefully acknowledge the support of NVIDIA Corporation with their donation of the TITAN X GPU used in this research. 


\section{References}

1. Bertalmio M, Sapiro G, Caselles V and Ballester C. Image inpainting. Proceedings of the 27th annual conference on Computer graphics and interactive techniques. 2000, 417-424.

2. Sdika, M., and Pelletier, D. Non rigid registration of multiple sclerosis brain images using lesion inpainting for morphometry or lesion mapping. Hum. Brain Mapp. 2009. 30, 10601067.

3. Guizard N, Nakamura K, Coupé P, Fonov V, Arnold D and Collins L. Non-Local Means Inpainting of MS Lesions in Longitudinal Image Processing. Front Neurosci. 2015; 9: 456.

4. Prados F, Cardoso MJ, Kanber B, et al. A multi-time-point modality-agnostic patch-based method for lesion filling in multiple sclerosis. Neuroimage. 2016;139:376-384.

5. Armanious K, Mecky Y, Gatidis S and Yang B. Adversarial inpainting of medical image modalisties. 2019. ICASSP2019.

6. Liu Y., Pan J., Su Z. Deep Blind Image Inpainting. Intelligence Science and Big Data Engineering. Visual Data Engineering. IScIDE 2019. Lecture Notes in Computer Science, vol 11935.

7. Coupé P., Tourdias T., Linck P., Romero J.E., Manjón J.V. LesionBrain: An Online Tool for White Matter Lesion Segmentation. Patch-Based Techniques in Medical Imaging. Patch-MI 2018. Lecture Notes in Computer Science, vol 11075.

8. Manjon, J.V., et al., Adaptive non-local means denoising of MR images with spatially varying noise levels. J Magn Reson Imaging, 2010. 31(1), 192-203.

9. Avants, B.B., et al., A reproducible evaluation of ANTs similarity metric performance in brain image registration. Neuroimage, 2011. 54(3), 2033-2044.

10. Tustison, N.J., et al., N4ITK: improved N3 bias correction. IEEE Trans Med Imaging, 2010. 29(6), 1310-20.

11. Ronneberger, O, Fischer P and Brox, T. U-Net: Convolutional Networks for Biomedical Image Segmentation. LNCS. 9351. 234-241.

12. Dou, Q., Yu, L., Chen, H., Jin, Y., Yang, X., Qin, J., Heng, P.A. 3D deeply supervised network for automated segmentation of volumetric medical images. Med. Image Anal. 2017. 41, 40-54.

13. Ashburner J. SPM: a history. Neuroimage. 2012. 62(2),791-800. 